
\title{
$\begin{array}{ll}\text { Research Square } & \begin{array}{l}\text { Preprints are preliminary reports that have not undergone peer review. } \\ \text { They should not be considered conclusive, used to inform clinical practice, } \\ \text { or referenced by the media as validated information. }\end{array}\end{array}$
}

\section{The Efficacy of Transdiagnostic Cognitive Behavioral Therapy on Migraine Headache: A Randomized Clinical Trial}

\author{
Forouzandeh Soleimanian-Boroujeni \\ Islamic Azad University \\ Negin Badihian \\ Isfahan University of Medical Sciences \\ Shervin Badihian \\ Johns Hopkins University School of Medicine \\ Vahid Shaygannejad ( $\sim$ Shaygannejad@med.mui.ac.ir) \\ Isfahan University of Medical Sciences \\ Yousef Gorji \\ Islamic Azad University
}

\section{Research Article}

Keywords: Cognitive Behavioral Therapy, Migraine Disorders, Headache, Clinical trial, Transdiagnostic therapy

Posted Date: January 3rd, 2022

DOI: https://doi.org/10.21203/rs.3.rs-1143097/v1

License: () (1) This work is licensed under a Creative Commons Attribution 4.0 International License. Read Full License 


\section{Abstract}

Introduction: Psychological interventions are shown to be effective in migraine, but not utilized routinely yet. We aimed to evaluate the efficacy of transdiagnostic cognitive behavioral therapy (TCBT) on people with migraine (PwM).

Method: This study was conducted on 40 PwM aged 20-50 years. We randomly assigned participants to two groups of intervention, receiving 10 sessions of TCBT, and control. Days with headache, headache severity, migraine-related disability and effects on daily life, number of pain-relivers taken for headache, depression, and anxiety were assessed pre-intervention, post-intervention (three-month followup), and one-month after TCBT termination (four-month follow-up).

Results: Thirty-five participants suffering moderate to severe migraine completed the study (16 and 19 in TCBT and control groups, respectively). TCBT improved all measured items between study time-points $(p<0.05)$ in the intervention group, while such an improvement was not observed in the control group. Between group comparisons revealed superiority of TCBT group compared to the control group in most measured items at three- and four-month follow-ups $(p<0.05)$.

Conclusion: Ten sessions of TCBT improved migraine severity, associated disability, anxiety, and depression in PwM, with persistent effects after one month of therapy termination. TCBT is an affordable, practical, and feasible intervention to be utilized for PwM.

Protocol registration: The study protocol was registered in clinicaltrial.gov (NCT03701477) prior to enrollment.

\section{Introduction}

Migraine is among the most prevalent primary headache disorders and one of the most important causes of disability worldwide [1]. It presents with recurrent attacks of moderate to severe headache lasting 4-72 hours. The global prevalence of migraine has been reported to be $11.6 \%$ in 2017 [2]. Migraine can affect both private and social aspects of life and lead to decreased quality of life. It mostly affects young to middle age population, hence, brings significant direct and indirect costs and is an important economic burden to the society. In 2016, the annual costs of migraine per person in the United States, due to using healthcare resources and productivity losses, were reported to be around $\$ 8,243$ and $\$ 2,649$ for chronic and episodic migraines, respectively [3]. The prevalence of psychological conditions, such as depression, anxiety, and mood disorders, is shown to be higher in people with migraine (PwM). These psychological conditions exacerbate the disability caused by migraine and would also increase the risk of progression to chronic form of the disease $[4,5]$. Current therapeutic approaches for migraine treatment mostly include using medications for prophylaxis and acute attacks. Regardless of the side effects associated with these medications, yet many PwM suffer from recurrent attacks even with regular usage of prophylactic medications [6]. Moreover, adherence to prophylactic migraine medications is shown to be low, mostly because of the side effects $[7,8]$. This could further deteriorate the condition and associated disability.

Some investigators have suggested migraine as a biopsychosocial condition that would respond well to psychological interventions at various stages [9]. A systematic review conducted in 2016 showed the efficacy of psychological interventions in migraine [10]. These psychological interventions include cognitive-behavioral therapy (CBT), relaxation trainings, stress management, massage therapy, etc. [9, 11]. CBT is reported to be efficacious in decreasing the frequency and severity of headaches in PwM [10,11]. However, delivering CBT faces multiple challenges: high expenses, presence of multiple comorbidities that can confuse both the therapist and the patient, inadequate resources, and limited number of trained psychologists in these techniques. Therefore, diagnostic-based psychological interventions such as CBT are less feasible and practical in the real world, despite being efficacious [10-12].

To address the feasibility and practicability issues, Transdiagnostic CBT (TCBT) could be considered. TCBT is a technique that focuses on therapeutic modules instead of constant similar therapeutic models. In this approach, the shared underlying mechanisms across diseases are addressed $[13,14]$. When delivering TCBT, the therapist does not need to focus on a specific psychological diagnosis and can implement a predetermined protocol for various psychological complaints. This means easier training of therapists and more resources for care delivery. A study has shown the effectiveness of TCBT on improving headache and psychological disturbances (e.g., anxiety and depression) among adolescents. [15]. No studies have evaluated the efficacy of TCBT on adult population with migraine, hitherto. Considering the high prevalence of comorbid anxiety and depressive symptoms among PwM and the efficacy of TCBT in these psychological conditions and headache in adolescents, we aimed to investigate the efficacy of TCBT on headache and the accompanying symptoms in a group of adult PwM. We hypothesized that TCBT can effectively improve migraine and decrease the associated disability and impact on PwM's lives.

\section{Materials And Method}




\section{Study Participants}

This is a randomized clinical trial conducted during October 2018 to April 2019 at a single outpatient private counselling clinic in Isfahan, Iran. The inclusion criteria were: 1) The diagnosis of migraine headache by the primary neurologist based on the criteria defined by International Classification of Headache Disorders III Beta (ICHD-III Beta) [16]; 2) Diagnosed with migraine at least 6 months prior to the enrollment; 3) High school graduate or higher level of education (in order to incorporate trainings during therapy); 4) Aged 20-50 years. Patients were excluded from the study in case of medication overuse diagnosed based on the criteria defined by ICHD-III Beta (taking nonsteroidal anti-inflammatory drugs or other pain relievers at least 15 days in each month; taking triptans or similar drugs at least 10 days each month for 3 months or more) [16]; using new prophylactic migraine medication during the study; suffering from other types of headache disorders in addition to migraine (e.g. cluster headaches, headaches due to structural abnormalities, etc.); undergoing any other psychological therapies during the study (not considering psychiatric medications that are used for migraine); and altered cognitive or mental health status (like dementia). Patients were initially recruited in the study neurologist's office, and then referred to the study team for further evaluations. The study procedure was explained to the participants and written informed consents were obtained from all participants. With a power of $80 \%$, a of 0.05 , and effect size of 0.9 , we estimated 16 participants are needed in each group to show a significant effect of the intervention. The effect size was estimated using HIT scores in the study by Sharma et al [15]. However, we decided to recruit 20 participants in each group given the possibility of dropouts or withdrawals. The study was approved by the ethics committee of Islamic Azad University of Khomeinishahr (ID:18820701962055) and was registered in clinicaltrials.gov prior to enrollment (ID: NCT03701477).

\section{Study design}

After obtaining informed consents and prior to randomization, we asked all participants to fill out the study questionnaires, explained below under "Outcome measures". We used random allocation software in four blocks to generate random numbers and divided participants into study groups. The intervention group underwent 10 sessions of TCBT (Table 1). The control group were scheduled for a single 3-hour meeting in which basic techniques of relaxation and stress management were discussed. All patients were also encouraged to adhere to their current migraine treatment and not to start new medications or therapies or discontinue current regimen, unless advised by their neurologist.

One week after the last therapy session of the intervention group, we asked the participants to fill out the same questionnaires for the second time as post-intervention measurement (3-month follow-up). In order to assess persistence of the effects of therapy sessions, we asked patients to come back one month after the last therapy session and complete the questionnaires for the third time (4-month followup). The control group completed the questionnaires on similar time-points. The primary investigators and outcome assessor were masked to the randomization status.

\section{Intervention}

The therapy protocol was derived from the suggested protocol by Sharma et al. on TCBT intervention for headache in adolescents [17]. We conducted 10 sessions of group based TCBT, each session lasting for 2 hours, by a trained, experienced psychologist. The sessions were held regularly at a definite time and place for continuous weeks. There was an exception for the last session which was held with a 2-week gap to give patients some extra time to incorporate what they have learned into their problems. We gave specific homework papers to the participants after each session. The homework included pre-specified tasks to practice what has been discussed during that session, practices to incorporate the new skills they have learned, and using these skills to deal with their problems. Patients were asked to do their homework and return it the next session. At the beginning of each therapy session, participants' homework papers were reviewed by the therapist and new tasks and skills were explained to the participants. At the end of each session, patients were asked to summarize the new skills they have learned and provide feedback on the new skills. Detailed description of topics and skills discussed in each session is presented in Table 1. 


\section{First session}

\section{Therapeutic agreement and psychoeducation}

1. Forming a therapeutic agreement between patients and the therapist regarding number of therapy sessions and their durations, the process of treatment, and confidentiality issues.

2. Educating patients about the nature and causes of headaches and its comorbid psychological disorders and discussing definition of anxiety.

3. Explaining the importance of completing homework at home, and providing instructions on homework,

4. Giving homework to patients on self-monitoring (recording dates of experiencing headaches, duration of the pain, and its severity based on a scale from 0 to 10; rating the average daily anxiety level on a scale of 0 to 100).

5. Asking patients to provide a list of situations that are associated with distressing anxiety for them.

6. Briefly explaining the content of the next session.

\section{Second session}

\section{Educating relaxation training and hierarchy of behavioral avoidance}

1. Educating relaxation techniques and preparation of hierarchy of behavioral avoidance for the better management of headache and anxiety responses.

2. Evaluating patients' homework on self-monitoring and analyzing headache triggers.

3. Explaining the components of anxiety to patients.

4. Giving homework to patients on relaxation exercises.

5. Giving homework to patients on self-monitoring.

6. Briefly explaining the content of the next session.

\section{Third session}

\section{Automatic negative thoughts and guided imagery}

1. Evaluating patients' problems in practicing relaxation techniques and preparing a hierarchy of behavioral problems as an important part of the treatment.

2. Discussing the importance of thoughts and educating how to identify automatic negative thoughts.

3. Giving homework to patients on recognizing and recording automatic negative thoughts.

4. Evaluating headache and anxiety daily diary recorded by the patient.

5. Delivering a CD containing audio on guided imaginary to patients to practice with at home.

6. Briefly explaining the content of the next session.

\section{Fourth session}

\section{Cognitive restructuring}

1. Ensuring that the patients have performed their self-monitoring homework and relaxation exercises.

2. Assessing automatic thoughts identified by patients during the past week.

3. Educating patients on how to challenge automatic thoughts.

4. Educating patients on cognitive errors and helping them to identify such errors.

5. Addressing patients' ambiguities.

6. Briefly explaining the content of the next session.

\section{Fifth session}

\section{Problem solving skills and distraction technique}

1. Evaluating patients' improvements in performing homework.

2. Educating problem solving skill and using role-playing techniques. 
3. Educating distraction technique to reduce overthinking.

4. Giving homework on problem solving and distraction skills.

5. Briefly explaining the content of the next session.

\section{Sixth session}

\section{Exposure and cognitive restructuring}

1. Evaluating problem solving homework, assessing learned skills and the way patients apply them in their daily lives.

2. Educating self-directed exposure to patients and giving them homework on this skill.

3. Educating advanced cognitive reconstruction steps and encouraging patients to apply them in real life situations.

4. Extensive focusing on daily general stress level instead of targeting a special problem.

5. Summarizing the session and addressing patients' ambiguities.

6. Briefly explaining the content of the next session.

\section{Seventh, Eighth, and Ninth session}

\section{Advanced cognitive restructuring, stress management, assertiveness skills training, preparation for therapy termination}

1. Evaluating self-monitoring homework, exposure, and cognitive reconstruction of patients to ensure their proper learning and applying them by patients in their lives.

2. guiding patients in cognitive reconstruction processes.

3. Educating stress management, assertiveness skills, and the ability to say "No".

4. Advising patients to continue cognitive reconstruction homework, stress management techniques, and assertiveness skills in appropriate situations.

5. preparing patients for the last therapy session.

Tenth session

\section{Relapse prevention and therapy termination}

1. Evaluating patients' performances in stress management and assertiveness skills.

2. Encouraging patients to continue doing homework and rewarding themselves.

3. Educating patients to administer the learned therapeutic techniques in case of disease recurrence.

4.Summarizing previous sessions and getting feedback from patients on different therapy sessions and effectiveness of the learned techniques.

5. Discussing with patients about the new perspectives they need to learn about themselves and their problems.

6. Congratulating patients on completing therapy sessions and acknowledging their participation in the program.

\section{Outcome measures}

Sociodemographic characteristics of participants including age, sex, education, number of days with headache within the past 30 days, headache severity, and history of medications used for migraine headache (both prophylactic medications and pain relievers) were recorded at baseline. To collect data on days with headache and amount of used medications, we asked participants to write those down in a headache diary.

We assessed participants at three time-points: baseline (prior to intervention), at the end of the last therapy session (3-month follow-up), and after 1 month of therapy termination (4-month follow-up). In each time-point, patients were evaluated regarding the level of pain, migraine effects on the individual's function in different aspects of life, migraine disability, depression, and anxiety.

For the assessment of pain severity, we used Wong-Baker Faces Pain Rating Scale (WBFPRS), also known as visual analog scale (VAS) [18]. It is a well-known scale consisted of six faces that represent the effect of pain on emotion. This instrument could be scored from 0 (no pain at all) to 10 (severe pain). For using this instrument, we asked patients to show the face that could better describe them during pain. Headache Impact Test- 6 (HIT-6) was used to assess migraine effects on the individual's function [19]. HIT-6 includes 6 questions 
evaluating different aspects of patient's life that may be affected by migraine. Each question is scored through Likert system and can be scored from 6 (never) to 13 (always). The total score of the questionnaire would be calculated by summing up scores from each single question. Higher scores indicate more severe migraine and scores over 50 show migraine has notably disturbed patient's daily activity [20]. The study was powered to HIT-6 as the primary outcome measure.

Migraine Disability Assessment Scale (MIDAS) was used to determine number of days patients have lost during the past 3 months due to the disability caused by migraine [21]. It contains 7 questions and by summing up the number of lost days written by the patient for 5 of the question, the disability would be assessed. Using this questionnaire, patient are classified to have no/very little disability (0-5), mild disability (6-10), moderate disability (11-20), and sever disability ( $\geq 21)$ [22]. The other 2 items are known as MIDAS-A and MIDAS-B and assess headache frequency (number of days spent with migraine headache during the past 3 months), and the average pain intensity (on a scale of 1-10), respectively. These 2 items are not accounted in measuring the total MIDAS score.

Hospital Anxiety and Depression Scale (HADS) was used to evaluate the level of anxiety and depression [23]. This scale contains 7 questions on anxiety and 7 on depression. Each question can be scored from 0 to 3 based on a Likert system. The total score for anxiety and depression is calculated by adding up the scores of each single question. This scale classifies patients to 3 different categories of normal, borderline, and abnormal anxiety and/or depression [24]. We used the validated Persian version of all these instruments, as cited above.

\section{Statistical analysis}

We described the results using mean values \pm standard deviation (SD) or frequencies (\%). Independent t-test or the non-parametric equal were used to compare baseline interval characteristics between two groups. We used Chi-square test to compare categorical data between two groups. Primary and secondary outcomes were assessed in each group using repeated measure analysis of variance (ANOVA). We used Greenhouse-Geisser correction in case sphericity was violated. The sphericity was evaluated using Mauchly's test of sphericity. In case of any statistically significant findings, we used post-hoc Bonferroni test to distinguish where the difference is. Prior to repeated measure ANOVA, Pearson correlation was used to identify possible covariates to be included in the analysis. We also compared the outcome measures as categorical data between two groups and in each group. The categories are previously explained under "outcome measures". Comparing these categorical outcome measures between groups was done using Chi-square test. To compare the categories in each single group between different time-points, we used Friedman test. To compare the outcome measures between two groups in each time-point separately, we used one-way analysis of covariance (ANCOVA), adjusting for the amounts reported in the baseline visit. For example, scores at three- and four-month follow-up were compared between two groups adjusting for the baseline scores. We used Statistical Package for Social Sciences software (SPSS, Armonk, NY; version 25) for statistical analysis and a p-value of less than 0.05 was considered as statistically significant.

\section{Results}

\section{Recruitment and baseline data}

We approached 87 patients, 18 patients were not eligible for the study (12 patients had educations under high school diploma, 6 were receiving psychological therapy) and 29 patients did not agree to participate in the study. Hence, 40 patients were enrolled and randomly assigned to intervention (TCBT) or control groups. We lost 4 patients in the intervention group before the 3-month follow-up, due to time limitations and not being able to maintain therapy. One patient in the control group did not show up for 3-month and 4-month follow-ups. Figure 1 shows the study diagram. Table 2 presents baseline characteristics of two study groups. The mean age of patients in the intervention and control group were $37.1 \pm 9.7$ and $37.3 \pm 9.2$, respectively $(p=0.937)$. Female patients comprised $93.8 \%(15$ cases $)$ of the intervention group and $89.5 \%$ (17 cases) of the control group ( $\mathrm{p}=0.653)$. The mean migraine duration was $16.2 \pm 10.9$ years in the intervention group and $9.3 \pm 8.1$ years in the control group $(p=0.063)$. 


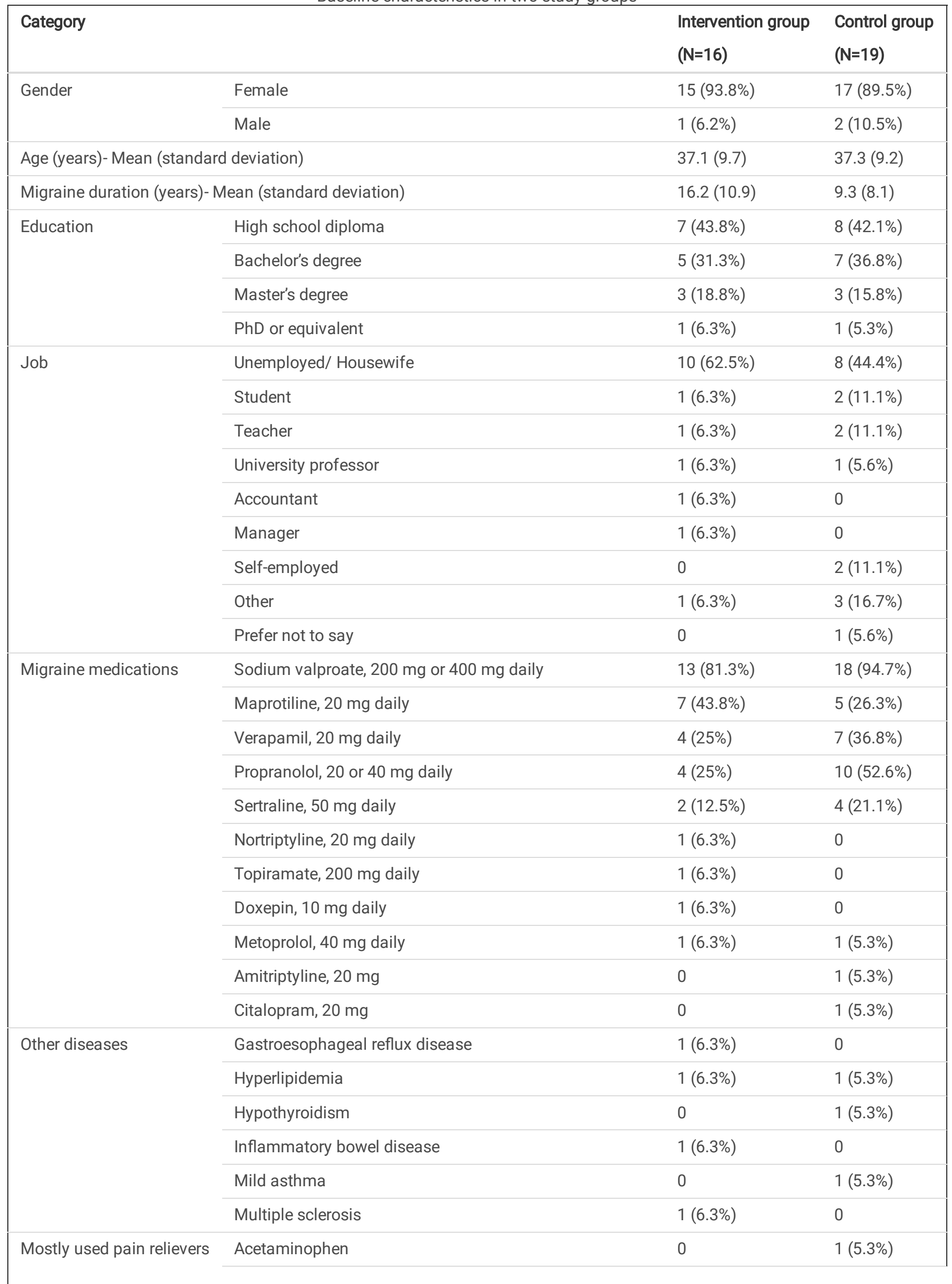




\begin{tabular}{|lll|}
\hline Category & $\begin{array}{l}\text { Intervention group } \\
\mathbf{( N = 1 6 )}\end{array}$ & $\begin{array}{l}\text { Control group } \\
\mathbf{( N = 1 9 )}\end{array}$ \\
\hline Diclofenac (supp) & $1(6.3 \%)$ & $2(10.5 \%)$ \\
\hline Ibuprofen (tab) & $6(37.5 \%)$ & $9(47.4 \%)$ \\
\hline Ibuprofen (cap) & 0 & $6(31.6 \%)$ \\
\hline Mixed pain relievers (acetaminophen, ibuprofen, caffeine) & $9(56.3 \%)$ & 0 \\
\hline
\end{tabular}

\section{Between group comparisons}

There were no statistically significant differences in frequency of categories of MIDAS, HIT, HADS-anxiety, and HADS-depression between two study groups at baseline $(p=0.797, p=0.352, p=0.959$, and $p=0.511$, respectively).

We used repeated measure ANOVA test to assess any interaction between assigned study group and observed outcomes. We found a statistically significant interaction between type of intervention and the scores/values from VAS, MIDAS (total, A, and B), HIT, HADS (both anxiety and depression), days with headache within the past 30 days, and number of pain-relivers taken during the past 30 days $(p<0.05)$. Figure 2 illustrates the comparison graphs on these outcome measures.

Moreover, we compared the outcome measures, both in numbers and in categories, between two groups using ANCOVA and Chi-square, as appropriate. These results are presented in Table 3. As demonstrated in the table, we observed improvement in days with headache, number of pain-relievers taken, VAS, MIDAS (total, A, and B) scores, and HADS (both anxiety and depression) scores immediately after intervention. However, we found no statistically significant difference in MIDAS categories, HIT scores and categories, and HADS anxiety categories between two groups at this time-point. On four-month follow-up, all these outcome measures showed a statistically significant difference between two groups, when adjusting for baseline scores. 
Table 3

Mean scores from study instruments and outcome measures in three study time-points

\begin{tabular}{|c|c|c|c|c|c|c|c|c|c|c|}
\hline \multicolumn{2}{|l|}{ Category } & \multicolumn{3}{|l|}{ Baseline } & \multicolumn{3}{|c|}{ 3-month follow-up } & \multicolumn{3}{|c|}{ 4-month follow-up } \\
\hline & & \multirow{3}{*}{$\begin{array}{l}\text { Intervention } \\
(\mathrm{N}-16) \\
\text { Mean (SD)/ } \\
\mathrm{N}(\%)\end{array}$} & \multirow{3}{*}{$\begin{array}{l}\text { Control } \\
(\mathrm{N}=19) \\
\text { Mean } \\
(\mathrm{SD}) / \\
\mathrm{N}(\%)\end{array}$} & \multirow[t]{3}{*}{$\begin{array}{l}\text { P- } \\
\text { value }^{1}\end{array}$} & \multirow{3}{*}{$\begin{array}{l}\text { Intervention } \\
(\mathrm{N}-16) \\
\text { Mean (SD)/ } \\
\mathrm{N}(\%)\end{array}$} & \multirow{3}{*}{$\begin{array}{l}\text { Control } \\
(\mathrm{N}=19) \\
\text { Mean } \\
(\mathrm{SD}) / \\
\mathrm{N}(\%)\end{array}$} & \multirow[t]{3}{*}{$\begin{array}{l}\text { P- } \\
\text { value }^{2}\end{array}$} & \multirow{3}{*}{$\begin{array}{l}\text { Intervention } \\
(\mathrm{N}-16) \\
\text { Mean (SD)/ } \\
\mathrm{N}(\%)\end{array}$} & \multirow{3}{*}{$\begin{array}{l}\text { Control } \\
(\mathrm{N}=19) \\
\text { Mean } \\
(\mathrm{SD}) / \\
\mathrm{N}(\%)\end{array}$} & \multirow[t]{3}{*}{$\begin{array}{l}\text { P- } \\
\text { value }^{2}\end{array}$} \\
\hline & & & & & & & & & & \\
\hline & & & & & & & & & & \\
\hline \multicolumn{2}{|c|}{$\begin{array}{l}\text { Days with headache } \\
\text { (days) }\end{array}$} & $13.5(6.1)$ & $\begin{array}{l}12.4 \\
(7.4)\end{array}$ & 0.341 & $7.9(4.3)$ & $\begin{array}{l}13.6 \\
(8.1)\end{array}$ & $0.001^{*}$ & $5.4(2.8)$ & $13.2(8)$ & $<0.001^{*}$ \\
\hline \multicolumn{2}{|c|}{ Pain-relievers (number) } & $13.6(6.7)$ & $\begin{array}{l}10 \\
(5.2)\end{array}$ & 0.080 & $6.4(4.6)$ & $\begin{array}{l}11.2 \\
(6.9)\end{array}$ & $<0.001^{\star}$ & $4.9(3.1)$ & $\begin{array}{l}11.4 \\
(7.1)\end{array}$ & $<0.001^{*}$ \\
\hline \multicolumn{2}{|c|}{ Headache severity-VAS } & $8.6(1.4)$ & $\begin{array}{l}8.9 \\
(1.2)\end{array}$ & 0.603 & $6.8(1.6)$ & $\begin{array}{l}8.8 \\
(1.3)\end{array}$ & $<0.001^{\star}$ & $6.1(1.4)$ & $\begin{array}{l}8.8 \\
(1.3)\end{array}$ & $<0.001^{*}$ \\
\hline \multicolumn{2}{|c|}{ MIDAS-total score } & $39.8(19.4)$ & $\begin{array}{l}34 \\
(23.7)\end{array}$ & 0.174 & $17.9(15.9)$ & $\begin{array}{l}36 \\
(24.8)\end{array}$ & $<0.001^{*}$ & $16(16.5)$ & $\begin{array}{l}36.4 \\
(25.1)\end{array}$ & $<0.001^{*}$ \\
\hline \multicolumn{2}{|l|}{ MIDAS-A } & $39.4(17.1)$ & $\begin{array}{l}35.3 \\
(19.6)\end{array}$ & 0.517 & $22.4(14.2)$ & $\begin{array}{l}37.8 \\
(22.2)\end{array}$ & $<0.001^{\star}$ & $19.8(13.6)$ & $\begin{array}{l}39.4 \\
(21.1)\end{array}$ & $<0.001^{*}$ \\
\hline \multicolumn{2}{|l|}{ MIDAS-B } & $7.9(1.6)$ & $\begin{array}{l}8.2 \\
(1.2)\end{array}$ & 0.494 & $6.3(1.4)$ & $8.2(1)$ & $<0.001^{\star}$ & $5.8(1.4)$ & $\begin{array}{l}8.2 \\
(1.1)\end{array}$ & $<0.001^{*}$ \\
\hline \multirow[t]{4}{*}{$\begin{array}{l}\text { MIDAS } \\
\text { category }\end{array}$} & $\begin{array}{l}\text { No } \\
\text { disability }\end{array}$ & 0 & 0 & 0.797 & $2(12.5 \%)$ & 0 & 0.106 & $4(25 \%)$ & 0 & $0.021^{\star}$ \\
\hline & Mild & $2(12.5 \%)$ & $\begin{array}{l}4 \\
(21.1 \%)\end{array}$ & & $5(31.3 \%)$ & $\begin{array}{l}4 \\
(21.1 \%)\end{array}$ & & $4(25 \%)$ & $\begin{array}{l}4 \\
(21.1 \%)\end{array}$ & \\
\hline & Moderate & $2(12.5 \%)$ & $\begin{array}{l}2 \\
(10.5 \%)\end{array}$ & & $4(25 \%)$ & $\begin{array}{l}2 \\
(10.5 \%)\end{array}$ & & $5(31.3 \%)$ & $\begin{array}{l}3 \\
(15 / 8 \%)\end{array}$ & \\
\hline & Severe & $12(75 \%)$ & $\begin{array}{l}13 \\
(68.4 \%)\end{array}$ & & $5(31.3 \%)$ & $\begin{array}{l}13 \\
(68.4 \%)\end{array}$ & & $3(18.8 \%)$ & $\begin{array}{l}12 \\
(63.2 \%)\end{array}$ & \\
\hline \multicolumn{2}{|l|}{ HIT } & $63.7(5.5)$ & $\begin{array}{l}65.6 \\
(6.6)\end{array}$ & 0.357 & $56.9(6.9)$ & $\begin{array}{l}62.4 \\
(9.2)\end{array}$ & 0.099 & $53.4(6)$ & $\begin{array}{l}63.7 \\
(7.8)\end{array}$ & $<0.001^{*}$ \\
\hline \multirow[t]{2}{*}{$\begin{array}{l}\text { HIT } \\
\text { category }\end{array}$} & $\begin{array}{l}\text { Not } \\
\text { severe }\end{array}$ & 0 & $\begin{array}{l}1 \\
(5.3 \%)\end{array}$ & 0.352 & $4(25 \%)$ & $\begin{array}{l}3 \\
(15.8 \%)\end{array}$ & 0.497 & $8(50 \%)$ & $\begin{array}{l}1 \\
(5.3 \%)\end{array}$ & $0.003^{*}$ \\
\hline & Severe & $16(100 \%)$ & $\begin{array}{l}18 \\
(94.7 \%)\end{array}$ & & $12(75 \%)$ & $\begin{array}{l}16 \\
(84.2 \%)\end{array}$ & & $8(50 \%)$ & $\begin{array}{l}18 \\
(94.7 \%)\end{array}$ & \\
\hline \multicolumn{2}{|c|}{ HADS-anxiety } & $10.5(4.5)$ & $\begin{array}{l}10.5 \\
(3.2)\end{array}$ & 0.642 & $7.9(4.3)$ & $\begin{array}{l}10.6 \\
(3.7)\end{array}$ & $0.002^{*}$ & $6.8(3.6)$ & $11.1(3)$ & $<0.001^{*}$ \\
\hline \multirow{3}{*}{$\begin{array}{l}\text { HADS- } \\
\text { anxiety } \\
\text { category }\end{array}$} & Normal & $4(25 \%)$ & $\begin{array}{l}4 \\
(21.1 \%)\end{array}$ & 0.959 & $10(62.5 \%)$ & $\begin{array}{l}6 \\
(31.6 \%)\end{array}$ & 0.092 & $10(62.5 \%)$ & $\begin{array}{l}3 \\
(15.8 \%)\end{array}$ & $0.013^{\star}$ \\
\hline & Borderline & $5(31.3 \%)$ & $\begin{array}{l}6 \\
(31.6 \%)\end{array}$ & & 0 & $\begin{array}{l}3 \\
(15.8 \%)\end{array}$ & & $3(18.8 \%)$ & $\begin{array}{l}5 \\
(26.3 \%)\end{array}$ & \\
\hline & Abnormal & $7(43.8 \%)$ & $\begin{array}{l}9 \\
(47.4 \%)\end{array}$ & & $6(37.5 \%)$ & $\begin{array}{l}10 \\
(52.6 \%)\end{array}$ & & $3(18.8 \%)$ & $\begin{array}{l}11 \\
(57.9 \%)\end{array}$ & \\
\hline \multicolumn{2}{|c|}{ HADS-depression } & $9.8(3.5)$ & $\begin{array}{l}10.3 \\
(3.7)\end{array}$ & 0.684 & $7.8(2.8)$ & $\begin{array}{l}10.6 \\
(2.9)\end{array}$ & $<0.001^{*}$ & $7.5(2.1)$ & $\begin{array}{l}11.2 \\
(3.2)\end{array}$ & $<0.001^{*}$ \\
\hline \multirow{3}{*}{$\begin{array}{l}\text { HADS- } \\
\text { depression } \\
\text { category }\end{array}$} & Normal & $5(31.3 \%)$ & $\begin{array}{l}6 \\
(31.6 \%)\end{array}$ & 0.511 & $10(62.5 \%)$ & $\begin{array}{l}3 \\
(15.8 \%)\end{array}$ & $0.017^{*}$ & $9(56.3 \%)$ & $\begin{array}{l}3 \\
(15.8 \%)\end{array}$ & $0.011^{\star}$ \\
\hline & Borderline & $5(31.3 \%)$ & $\begin{array}{l}3 \\
(15.8 \%)\end{array}$ & & $3(18.8 \%)$ & $\begin{array}{l}8 \\
(42.1 \%)\end{array}$ & & $5(31.3 \%)$ & $\begin{array}{l}5 \\
(26.3 \%)\end{array}$ & \\
\hline & Abnormal & $6(37.5 \%)$ & $\begin{array}{l}10 \\
(52.6 \%)\end{array}$ & & $3(18.8 \%)$ & $\begin{array}{l}8 \\
(42.1 \%)\end{array}$ & & $2(12.5 \%)$ & $\begin{array}{l}11 \\
(57.9 \%)\end{array}$ & \\
\hline
\end{tabular}




\begin{tabular}{|c|c|c|c|c|c|c|c|c|c|}
\hline \multirow[t]{3}{*}{ Category } & \multicolumn{3}{|l|}{ Baseline } & \multicolumn{3}{|c|}{ 3-month follow-up } & \multicolumn{3}{|c|}{ 4-month follow-up } \\
\hline & $\begin{array}{l}\text { Intervention } \\
(\mathrm{N}-16)\end{array}$ & $\begin{array}{l}\text { Control } \\
(\mathrm{N}=19)\end{array}$ & $\begin{array}{l}\text { P- } \\
\text { value }^{1}\end{array}$ & $\begin{array}{l}\text { Intervention } \\
(\mathrm{N}-16)\end{array}$ & $\begin{array}{l}\text { Control } \\
(\mathrm{N}=19)\end{array}$ & $\begin{array}{l}\text { P- } \\
\text { value }^{2}\end{array}$ & $\begin{array}{l}\text { Intervention } \\
(\mathrm{N}-16)\end{array}$ & $\begin{array}{l}\text { Control } \\
(\mathrm{N}=19)\end{array}$ & $\begin{array}{l}\text { P- } \\
\text { value }^{2}\end{array}$ \\
\hline & $\begin{array}{l}\text { Mean (SD)/ } \\
\mathrm{N}(\%)\end{array}$ & $\begin{array}{l}\text { Mean } \\
(\text { SD)/ } \\
\mathrm{N}(\%)\end{array}$ & & $\begin{array}{l}\text { Mean (SD)/ } \\
\mathrm{N}(\%)\end{array}$ & $\begin{array}{l}\text { Mean } \\
\text { (SD)/ } \\
\mathrm{N}(\%)\end{array}$ & & $\begin{array}{l}\text { Mean (SD)/ } \\
\mathrm{N}(\%)\end{array}$ & $\begin{array}{l}\text { Mean } \\
\text { (SD)/ } \\
\mathrm{N}(\%)\end{array}$ & \\
\hline $\begin{array}{l}\text { Emergency department } \\
\text { visits for headache per } \\
\text { month (number of } \\
\text { cases) }\end{array}$ & $2(12.5 \%)$ & $\begin{array}{l}3 \\
(15.8 \%)\end{array}$ & 0.782 & $1(6.3 \%)$ & $\begin{array}{l}1 \\
(5.3 \%)\end{array}$ & 0.900 & 0 & $\begin{array}{l}1 \\
(5.3 \%)\end{array}$ & 0.352 \\
\hline \multicolumn{10}{|c|}{$\begin{array}{l}\text { VAS: Visual Analog Scale; MIDAS: Migraine Disability Assessment Scale; HIT: Headache Impact Test; HADS: Hospital Anxiety and } \\
\text { Depression Scale; Days: Number of days the patients have experienced headache within the past } 30 \text { days; Pain relievers: Number of } \\
\text { pain-relievers taken during the past } 30 \text { days. } \\
{ }^{1} \text { Between group comparison. P-value calculated using Chi-square test (for categorical variables) or independent t-test (for interval } \\
\text { variables) as appropriate. }\end{array}$} \\
\hline
\end{tabular}

\section{In-group comparisons}

We used repeated measure ANOVA test to evaluate changes in outcome measures and compare the values between different study timepoints. Based on these findings, we observed statistically significant differences in all categories (VAS, MIDAS, HIT, HADS, headache days, and pain-relievers) between study time-points in the intervention group $(p<0.05)$. However, we found no similar statistically significant differences in the control group $(p>0.05)$.

Post-hoc analysis of the results in the intervention group using Bonferroni test is presented in Table 4. According to the table, there were statistically significant differences between the baseline scores/values and those scores from 3-month and 4-month follow-ups in all outcome measures $(p<0.05)$. Moreover, no statistically significant differences were found between 3-month and 4-month follow-ups in MIDAS-B, HADS-depression, and number of pain-relievers ( $p>0.05)$, indicating a persistent effect of the intervention. Other outcome measures showed continued declined scores in the 4-month follow-up with statistically significant differences compared to the 3-month follow-up scores $(p<0.05)$, indicating persistent improvement. 
Table 4

Pairwise comparison of outcome measures between different study time-points in the intervention group.

\begin{tabular}{|c|c|c|c|c|c|c|}
\hline Category & $\begin{array}{l}\text { Time- } \\
\text { point }\end{array}$ & $\begin{array}{l}\text { Reference } \\
\text { time-point }\end{array}$ & $\begin{array}{l}\text { Mean } \\
\text { Difference }\end{array}$ & $\begin{array}{l}\text { Standard } \\
\text { Error }\end{array}$ & $\begin{array}{l}\mathrm{P}- \\
\text { value }\end{array}$ & $\begin{array}{l}95 \% \text { Confidence Interval } \\
\text { for Difference }\end{array}$ \\
\hline \multirow{3}{*}{$\begin{array}{l}\text { Visual analog scale (headache } \\
\text { severity) }\end{array}$} & \multirow[t]{2}{*}{1} & 2 & 1.875 & 0.340 & $<0.001$ & $(1.150,2.600)$ \\
\hline & & 3 & 2.500 & 0.342 & $<0.001$ & $(1.772,3.228)$ \\
\hline & 2 & 3 & 0.625 & 0.352 & 0.096 & $(-0.125,1.375)$ \\
\hline \multirow{3}{*}{$\begin{array}{l}\text { Migraine disability assessment scale } \\
\text { (MIDAS) }\end{array}$} & \multirow[t]{2}{*}{1} & 2 & 21.875 & 4.513 & $<0.001$ & $(12.256,31.494)$ \\
\hline & & 3 & 23.813 & 4.841 & $<0.001$ & $(13.494,34.131)$ \\
\hline & 2 & 3 & 1.938 & 0.692 & 0.013 & $(0.462,3.413)$ \\
\hline \multirow[t]{3}{*}{ MIDAS-A } & \multirow[t]{2}{*}{1} & 2 & 16.938 & 3.269 & $<0.001$ & $(9.970,23.905)$ \\
\hline & & 3 & 19.563 & 3.217 & $<0.001$ & $(12.705,26.420)$ \\
\hline & 2 & 3 & 2.625 & 1.197 & 0.044 & $(0.074,5.176)$ \\
\hline \multirow[t]{3}{*}{ MIDAS-B } & \multirow[t]{2}{*}{1} & 2 & 1.594 & 0.369 & 0.001 & $(0.808,2.379)$ \\
\hline & & 3 & 2.063 & 0.413 & $<0.001$ & $(1.182,2.943)$ \\
\hline & 2 & 3 & 0.469 & 0.314 & 0.157 & $(-0.202,1.139)$ \\
\hline \multirow[t]{3}{*}{ Headache impact test (HIT) } & \multirow[t]{2}{*}{1} & 2 & 6.750 & 1.493 & $<0.001$ & $(3.568,9.932)$ \\
\hline & & 3 & 10.313 & 1.128 & $<0.001$ & $(7.908,12.717)$ \\
\hline & 2 & 3 & 3.563 & 1.151 & 0.007 & $(1.109,6.016)$ \\
\hline \multirow{3}{*}{$\begin{array}{l}\text { Hospital anxiety and depression scale- } \\
\text { Anxiety }\end{array}$} & \multirow[t]{2}{*}{1} & 2 & 2.625 & 0.774 & 0.004 & $(0.975,4.275)$ \\
\hline & & 3 & 3.688 & 0.762 & $<0.001$ & $(2.063,5.312)$ \\
\hline & 2 & 3 & 1.063 & 0.403 & 0.019 & $(0.204,1.921)$ \\
\hline \multirow{3}{*}{$\begin{array}{l}\text { Hospital anxiety and depression scale- } \\
\text { Depression }\end{array}$} & \multirow[t]{2}{*}{1} & 2 & 2.063 & 0.433 & $<0.001$ & $(1.140,2.985)$ \\
\hline & & 3 & 2.313 & 0.561 & 0.001 & $(1.118,3.507)$ \\
\hline & 2 & 3 & 0.250 & 0.487 & 0.615 & $(-0.789,1.289)$ \\
\hline \multirow{3}{*}{$\begin{array}{l}\text { Days with headache within the past } 30 \\
\text { days }\end{array}$} & \multirow[t]{2}{*}{1} & 2 & 5.625 & 1.179 & $<0.001$ & $(3.111,8.139)$ \\
\hline & & 3 & 8.125 & 1.541 & $<0.001$ & $(4.841,11.409)$ \\
\hline & 2 & 3 & 2.500 & 1.114 & 0.040 & $(0.125,4.875)$ \\
\hline \multirow{3}{*}{$\begin{array}{l}\text { Number of pain-relievers taken during } \\
\text { the past } 30 \text { days }\end{array}$} & \multirow[t]{2}{*}{1} & 2 & 7.188 & 1.085 & $<0.001$ & $(4.875,9.500)$ \\
\hline & & 3 & 8.688 & 1.413 & $<0.001$ & $(5.675,11.700)$ \\
\hline & 2 & 3 & 1.500 & 0.764 & 0.068 & $(-0.128,3.128)$ \\
\hline
\end{tabular}

Comparing the categorized MIDAS, HIT, HADS-anxiety, and HADS-depression in the intervention group between three time-points showed statistically significant differences between these frequencies $(p<0.001, p=0.005, p=0.003$, and $p=0.005$, respectively). Similar analysis in the control group for MIDAS, HIT, HADS-anxiety, and HADS-depression showed no statistically significant differences $(p=0.368, p=0.264$, $\mathrm{p}=0.229$, and $\mathrm{p}=0.326$, respectively).

\section{Discussion}

In the present study, we investigated the efficacy of TCBT in PwM. TCBT showed favorable effects on decreasing days of headache, headache severity, migraine related disability, migraine effects on daily life, number of pain-relievers used for headache, depression, and 
anxiety. These effects were also present after one month of therapy discontinuation indicating persistency of the effects.

A systematic review of 24 studies showed the efficacy of psychological interventions in migraine [10]. Behavioral therapies with different approaches are among the most suggested psychological interventions for PwM [9]. Despite the observed efficacy in research setting, a gap in practice exists in delivering mental health services $[25,26]$. Multiple factors have been suggested to contribute to this gap, including cost, patient willingness to pursue psychological interventions, adherence to therapy, and availability of trained personnel. With regards to patient willingness, a recent study indicated tendency in pursuing behavioral therapy among PwM especially in those with moderate to severe level; however, patients were not willing to pay for the therapy sessions when they did not have insurance coverage for such treatments [12].

While in-person behavioral therapy sessions cost considerable amounts for both the patient and health care system, implementing groupbased sessions are less expensive and more practical. The transdiagnostic nature of TCBT means this technique can be used in a groupbased setting with individuals suffering from different types of disorders and comorbidities [14, 15, 27]. This would further facilitate the process for therapists and centers delivering this service to enroll enough number of cases for a group. Given these characteristics, TCBT is more favorable for both the patient and health care delivery system [13,27]. We designed the therapy modules within 10 sessions of two hours, as brief therapy modules have been suggested to increase feasibility of delivering care and patient adherence with favorable outcomes [28].

In the study by Sharma et al in 2017, 63 adolescents suffering concurrent anxiety and primary headache (episodic tension-type, migraine, or cluster headache) were divided into two groups of intervention and control. The intervention group underwent 12 weeks of group based TCBT while the control group only continued their previous pharmacotherapy. Improvements in the Headache Impact Test and Global Assessment Scale for Children were observed at the end of the study in both groups; however, results from the TCBT group were better than the control group [15]. Also, improvement in the State Trait Anxiety Inventory was only observed in the TCBT group and not the control group. Of note, the study was limited by different baseline features of two groups, favoring the intervention group [15].

In the study by Klan et al. the efficacy of an integrative CBT program was assessed among 9 adults suffering migraine [29]. The program consisted of 7 sessions, each lasting for 90 minutes. Patients reported a high satisfaction with therapy sessions, and the treatment integrity assessment and the qualitative interview by the patients were favorable as well. However, no statistically significant improvements in pre-treatment and post-treatment comparisons of the following tests were observed: The Headache Disability Inventory, The Pain Disability Index, Headache Impact Test, Depression Anxiety Stress Scales, Headache Management Self-Efficacy Scale-short form, Headache Triggers Sensitivity and Avoidance Questionnaire, and Chronic Pain Acceptance Questionnaire [29]. The small number of participants has possibly been the main reason for lack of improvement in observed scores.

In another study, Sajadinejad et al. investigated the efficacy of 9 weeks of group-based CBT (one session each week) on 20 female patients suffering tension type headache or migraine. Researchers used headache disability inventory and Beck depression inventory before intervention, after 5 weeks of therapy and at the last therapy session. They reported improvements in headache disability and depression in the assessments performed after 5 and 9 weeks of therapy. Worth mentioning that the authors failed to report headache characteristics and its associated changes among their patients [30]. Neither the study by Klan et al. nor the study by Sajadinejad et al had a control group for comparison.

In the current study, we found improved quantitative scores in all used questionnaires at the time of therapy termination (three-month follow-up), except for HIT score Also, the categorical MIDAS, HIT, and HADS-anxiety were not different between two groups, with only HADSdepression showing improvement. One month after therapy termination (four-month follow-up), all scores and categories of the mentioned outcome measures were improved in the intervention group compared to the control group. These findings indicate favorable outcome of TCBT among PwM in improving headache and associated disabilities, as well as concomitant anxiety and depression.

Regarding the persistency of behavioral therapy effects, it has been reported that the beneficial effects of CBT in patients suffering headaches and migraine would decline within several weeks of therapy termination [11]. Here we did not find such declines on the therapeutic effects of TCBT based on the assessments performed one month after therapy termination. In-group comparison of outcome measures in the intervention group showed no change or improvement of outcomes from three-month to four-month follow-up, implying the short-term persistency of our intervention.

To have a better understanding of our findings, we need to see the demographics of our recruited cases: young females with a graduate degree in more than half of cases, with severe migraine, severe associated disability, and abnormal levels of anxiety and depression in most cases. We believe these patients were more likely to participate in the study given the level of distress they are bearing, compared to

Page $12 / 17$ 
those with mild to moderate severity or disability and more controllable migraine. While our cases are probably not representative of all PwM, they show how effective TCBT could be in severe migraine cases. Moreover, anxiety and depression are known as precipitating factors for migraine that could lead to disease progression [31,32]. Therefore, interventions affecting these factors could ultimately lead to a better patient-reported outcome. We speculate that changes in patients' perspectives toward their daily lives and the accompanying anxieties have helped them to reconsider their habitude in dealing with problems. We think that TCBT have helped patients to strengthen their ability in dealing with their headache through new skills on controlling stress and coping with pain and distressing situations. Hence, their feelings of disability were significantly reduced, and they were able to implement the new learned skills in their lives, leading to the persistency of effects.

There are some limitations to the present study. First, most of our participants were young females. Considering that migraine is more prevalent among women and that women are more willing to pursue psychological interventions, this was inevitable in our recruitment process. Second, we were not able to follow up patients for more than one-month after therapy termination, given the limited funds and personnel. Therefore, we are not able to evaluate the long-term persistency of TCBT among PwM. Third, given the nature of our intervention, we were not able to mask patients or our therapist of the randomization status. We tried to reduce the placebo effect by holding a therapy session for the control group; but this probably would not eliminate the placebo effect completely considering the longer duration of therapy in the intervention group and their engagement with the therapy by working on their assignments. Fourth, we did not ask our patients to complete satisfaction surveys after each session of therapy. But they were asked to give verbal feedback at the end of each session, and they reported high levels of satisfaction. Despite these limitations, to the best of our knowledge this is the first study evaluating the efficacy of TCBT on adult PWM, compared to a control group receiving sham therapy session. Moreover, we provided a shortterm follow-up evaluation which has not been done in many of the previous studies. Future studies with larger sample sizes, a more diverse patient population, and longer follow-ups are needed to confirm our findings.

\section{Conclusion}

This study provides evidence on the efficacy of TCBT among PWM in lowering migraine severity, decreasing the associated disability, and improving anxiety and depression. It also shows that TCBT has short-term persistent benefits in this group of patients. TCBT is a is a feasible, practical, and cost-effective technique, and could be used along with the routine medication therapy in PwM.

\section{Clinical implications}

Transdiagnostic cognitive behavioral therapy (TCBT) improves migraine severity.

TCBT decreases migraine-associated disability and improves comorbid anxiety and depression in people with migraine.

TCBT is a feasible, practical, and cost-effective technique to be used in people with migraine.

\section{Public health relevance}

Despite the reported efficacy of psychological interventions in various medical conditions, these interventions are not widely used in real practice. Effective, brief interventions could facilitate the utilization of these techniques.

\section{Declarations}

\section{Funding}

This study was funded by Islamic Azad University of Khomeinishahr (ID: 18820701962055), granted to Dr. Yousef Gorji and Dr. Vahid Shaygannejad, as co-primary investigators of the study.

\section{Acknowledgement}

We would like to thank the Islamic Azad University of Khomeinishahr for supporting the study, Shokouh Counselling Center for providing the logistics for therapy sessions, and all the generous patients whose contribution made running this project successful.

\section{Data availability statement}

Anonymized data will be made available upon request from qualified investigators.

\section{Conflict of interest}


The authors report no conflict of interest.

\section{Author contribution}

F.S. contributed to designing the study, conducting the study, data collection, interpreting the findings, drafting the manuscript, and approving the final manuscript as submitted.

N.B. contributed to designing the study, data collection, interpreting the findings, drafting the manuscript, and approving the final manuscript as submitted.

S.B. contributed to designing the study, data analysis, interpreting the findings, drafting the manuscript, and approving the final manuscript as submitted.

V.S. contributed to conceptualizing the study, designing the study, interpreting the findings, and approving the final manuscript as submitted.

Y.G. contributed to conceptualizing the study, designing the study, interpreting the findings, and approving the final manuscript as submitted.

\section{References}

1. Stovner LJ, Nichols E, Steiner TJ, Abd-Allah F, Abdelalim A, Al-Raddadi RM, et al. Global, regional, and national burden of migraine and tension-type headache, 1990-2016: a systematic analysis for the Global Burden of Disease Study 2016. The Lancet Neurology. 2018;17 11:954-76; doi: 10.1016/s1474-4422(18)30322-3.

2. Woldeamanuel YW, Cowan RP. Migraine affects 1 in 10 people worldwide featuring recent rise: A systematic review and meta-analysis of community-based studies involving 6 million participants. J Neurol Sci. 2017;372:307-15; doi: 10.1016/j.jns.2016.11.071.

3. Messali A, Sanderson JC, Blumenfeld AM, Goadsby PJ, Buse DC, Varon SF, et al. Direct and Indirect Costs of Chronic and Episodic Migraine in the United States: A Web-Based Survey. Headache. 2016;56 2:306-22; doi: 10.1111/head.12755.

4. Buse DC, Silberstein SD, Manack AN, Papapetropoulos S, Lipton RB. Psychiatric comorbidities of episodic and chronic migraine. J Neurol. 2013;260 8:1960-9; doi: 10.1007/s00415-012-6725-x.

5. Bigal ME, Lipton RB. The epidemiology, burden, and comorbidities of migraine. Neurol Clin. 2009;27 2:321-34; doi: 10.1016/j.ncl.2008.11.011.

6. Ha H, Gonzalez A. Migraine Headache Prophylaxis. American family physician. 2019;99 1:17-24.

7. Hepp Z, Dodick DW, Varon SF, Gillard P, Hansen RN, Devine EB. Adherence to oral migraine-preventive medications among patients with chronic migraine. Cephalalgia. 2015;35 6:478-88.

8. Hepp Z, Bloudek LM, Varon SF. Systematic review of migraine prophylaxis adherence and persistence. J Manag Care Pharm. 2014;20 1:22-33; doi: 10.18553/jmcp.2014.20.1.22.

9. Singer AB, Buse DC, Seng EK. Behavioral treatments for migraine management: useful at each step of migraine care. Current neurology and neuroscience reports. 2015;15 4:14; doi: 10.1007/s11910-015-0533-5.

10. Sullivan A, Cousins S, Ridsdale L. Psychological interventions for migraine: a systematic review. J Neurol. 2016;263 12:2369-77; doi: $10.1007 / \mathrm{s} 00415-016-8126-z$.

11. Harris P, Loveman E, Clegg A, Easton S, Berry N. Systematic review of cognitive behavioural therapy for the management of headaches and migraines in adults. British journal of pain. 2015;9 4:213-24; doi: 10.1177/2049463715578291.

12. Minen MT, Jalloh A, Begasse de Dhaem O, Seng EK. Behavioral Therapy Preferences in People With Migraine. Headache. 2020;60 6:1093-102; doi: 10.1111/head.13790.

13. Bullis JR, Sauer-Zavala S, Bentley KH, Thompson-Hollands J, Carl JR, Barlow DH. The unified protocol for transdiagnostic treatment of emotional disorders: preliminary exploration of effectiveness for group delivery. Behavior modification. 2015;39 2:295-321; doi: $10.1177 / 0145445514553094$.

14. Harvey A, Watkins E, Mansell W, Shafran R. Cognitive Behavioural Processes across Psychological Disorders (DRAFT). Oxford University Press; 2004.

15. Sharma P, Mehta M, Sagar R. Efficacy of transdiagnostic cognitive-behavioral group therapy for anxiety disorders and headache in adolescents. Journal of anxiety disorders. 2017;46:78-84; doi: 10.1016/j.janxdis.2016.11.001.

16. Society HCCotIH. The international classification of headache disorders, (beta version). Cephalalgia. 2013;33 9:629-808.

Page 14/17 
17. Sharma P, Mehta M, Sagar R. Headache-A Transdiagnostic Approach. In: A Practical Approach to Cognitive Behaviour Therapy for Adolescents. Springer; 2015. p. 285-310.

18. Carlsson AM. Assessment of chronic pain. I. Aspects of the reliability and validity of the visual analogue scale. Pain. 1983;16 1:87-101; doi: 10.1016/0304-3959(83)90088-X.

19. Zandifar A, Banihashemi M, Haghdoost F, Masjedi SS, Manouchehri N, Asgari F, et al. Reliability and Validity of the Persian HIT-6 Questionnaire in Migraine and Tension-type Headache. Pain practice : the official journal of World Institute of Pain. 2014;14 7:625-31; doi: 10.1111/papr.12120.

20. Yang M, Rendas-Baum R, Varon SF, Kosinski M. Validation of the Headache Impact Test (HIT-6) across episodic and chronic migraine. Cephalalgia. 2011;31 3:357-67; doi: 10.1177/0333102410379890.

21. Zandifar A, Asgari F, Haghdoost F, Masjedi SS, Manouchehri N, Banihashemi M, et al. Reliability and validity of the migraine disability assessment scale among migraine and tension type headache in Iranian patients. BioMed research international. 2014;2014:978064; doi: $10.1155 / 2014 / 978064$.

22. Stewart WF, Lipton RB, Dowson AJ, Sawyer J. Development and testing of the Migraine Disability Assessment (MIDAS) Questionnaire to assess headache-related disability. Neurology. 2001;56 6 Suppl 1:S20-8; doi: 10.1212/wnl.56.suppl_1.s20.

23. Montazeri A, Vahdaninia M, Ebrahimi M, Jarvandi S. The Hospital Anxiety and Depression Scale (HADS): translation and validation study of the Iranian version. Health and quality of life outcomes. 2003;1:14; doi: 10.1186/1477-7525-1-14.

24. Bjelland I, Dahl AA, Haug TT, Neckelmann D. The validity of the Hospital Anxiety and Depression Scale. An updated literature review. Journal of psychosomatic research. 2002;52 2:69-77; doi: 10.1016/s0022-3999(01)00296-3.

25. Stirman SW, Gutner CA, Langdon K, Graham JR. Bridging the gap between research and practice in mental health service settings: An overview of developments in implementation theory and research. Behavior therapy. 2016;47 6:920-36.

26. Herschell AD, McNeil CB, McNeil DW. Clinical child psychology's progress in disseminating empirically supported treatments. Clinical Psychology: Science. 2004;11 3:267-88.

27. McEvoy PM, Nathan P, Norton PJ. Efficacy of Transdiagnostic Treatments: A Review of Published Outcome Studies and Future Research Directions. Journal of Cognitive Psychotherapy. 2009;23 1:20-33; doi: 10.1891/0889-8391.23.1.20.

28. Crawley SA, Kendall PC, Benjamin CL, Brodman DM, Wei C, Beidas RS, et al. Brief Cognitive-Behavioral Therapy for Anxious Youth: Feasibility and Initial Outcomes. Cogn Behav Pract. 2013;20 2:123-33; doi: 10.1016/j.cbpra.2012.07.003.

29. Klan T, Liesering-Latta E, Gaul C, Martin PR, Witthoft M. An Integrative Cognitive Behavioral Therapy Program for Adults With Migraine: A Feasibility Study. Headache. 2019;59 5:741-55; doi: 10.1111/head.13532.

30. Sajadinejad MS, Mohammadi N, Taghavi SMR, Ashgazadeh N. Therapeutic Effects of Cognitive-Behavioral Group Therapy on Headache Disability and Depression in Patients with Migraine and Tension Headache. Iranian Journal of Psychiatry and Clinical Psychology. 2009;14 4:411-8.

31. Bigal ME, Lipton RBJHTJoH, Pain F. Modifiable risk factors for migraine progression. 2006;46 9:1334-43.

32. Peroutka SJJCp, reports $h$. What turns on a migraine? A systematic review of migraine precipitating factors. 2014;18 10:454.

\section{Figures}




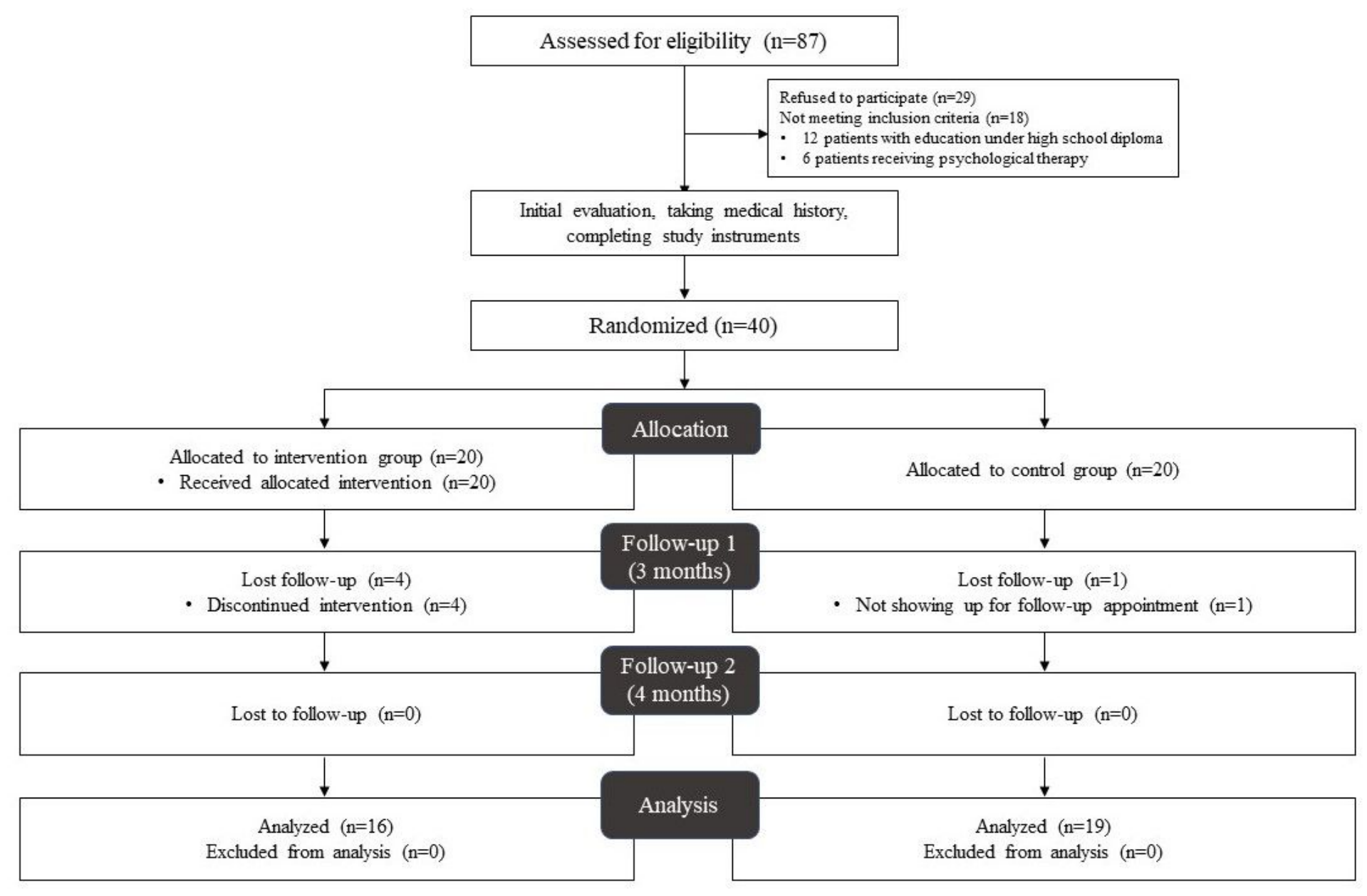

Figure 1

Study diagram 

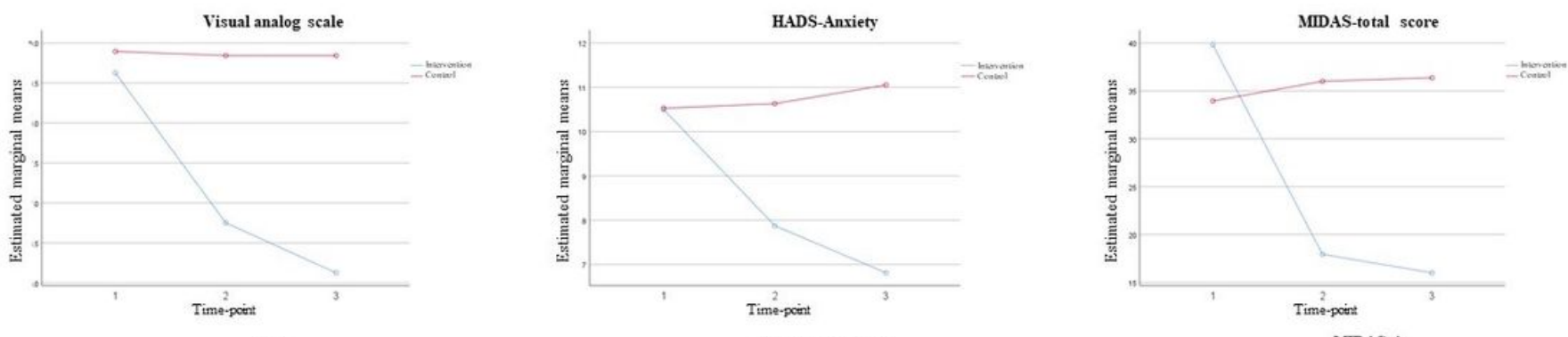

HIT
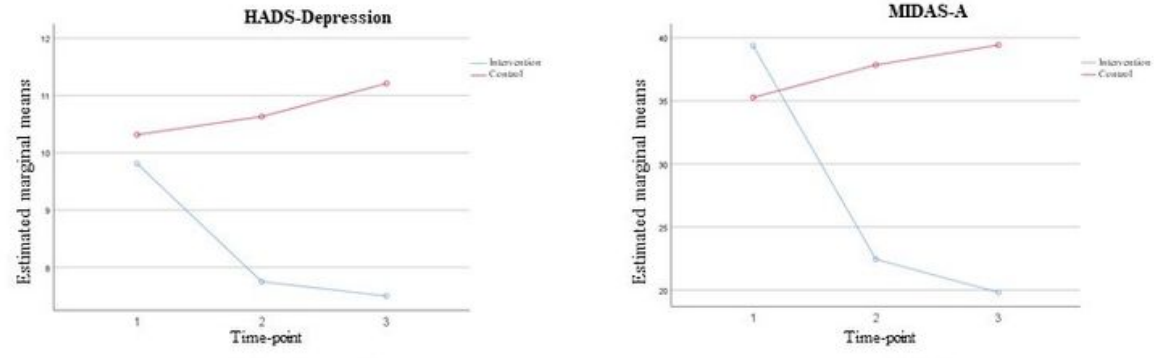

Days with headache
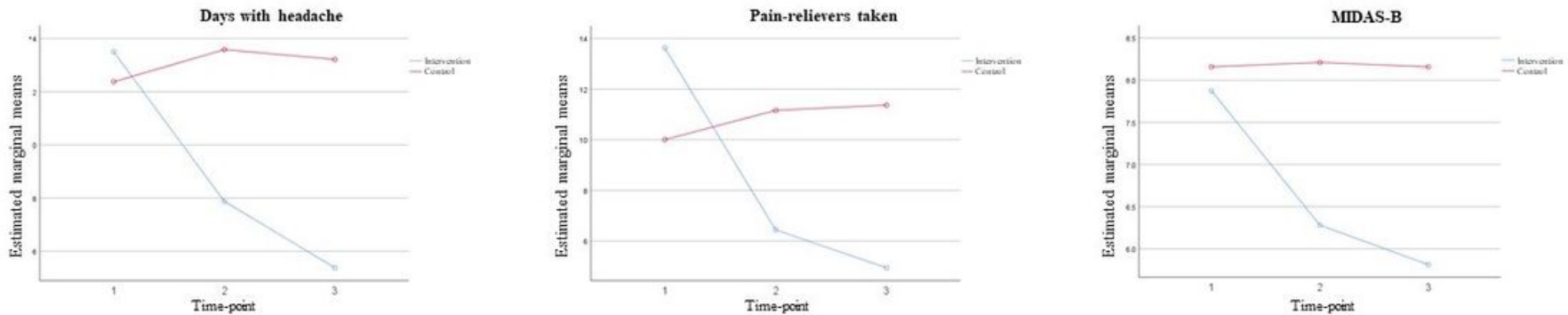

Figure 2

Comparison of mean scores from study instruments and outcome measures in three study time-points

HIT: Headache Impact Test; Days with headache: Number of days the patients has experienced headache within the past 30 days; Pain relievers taken: Number of pain-relievers taken during the past 30 days; HADS: Hospital Anxiety and Depression Scale; MIDAS: Migraine Disability Assessment Scale. Estimated marginal means evaluated using repeated measure analysis of variance. 